\title{
Non inductive formation of an extremely overdense spherical Tokamak by electron Bernstein wave heating and current drive on LATE
}

\author{
Masaki Uchida, a, Yuto Noguchii, Hitoshi Tanaka ${ }^{1}$, Takashi Maekawa ${ }^{1}$ \\ ${ }^{1}$ Graduate School of Energy Science, Kyoto University, 606-8502 Kyoto, Japan
}

\begin{abstract}
An extremely overdense special Tokamak plasma has been non-inductively formed and maintained by electron Bernstein (EB) wave heating and current drive in the Low Aspect ratio Torus Experiment (LATE) device. The plasma current reaches $12 \mathrm{kA}$ and the line-averaged electron density exceeds 7 times the plasma cut off density by injecting a $2.45 \mathrm{GHz}$ microwave power of $60 \mathrm{~kW}$. Such a highly overdense plasma is obtained when the upper hybrid resonance layer lies to the higher field side of the 2nd harmonic ECR layer, which may realize a good coupling to EB waves at their first propagation band. The effect of the injection polarization on the mode conversion rate to EB waves at the extremely overdense regime has been investigated and an improvement in the plasma current is observed.
\end{abstract}

\section{Introduction}

Recently there has been a considerable interest in noninductive start-up of Tokamak plasma without the use of the central solenoid (CS). If the plasma current can be non-inductively started up, the CS could be reduced or eliminated from the Tokamak device. These provide a major technical advantage for designing a future compact reactor, such as VECTOR [1] and Slim-CS [2]. For spherical Tokamak (ST) based devices such as CTF-ST [3] and FNSF-ST [4], elimination of CS is crucial since there is a severely restricted space in the centre column of ST to hold an aspect ratio sufficiently low. Therefore the establishment of a non-inductive start-up method is necessary for future ST-based fusion devices.

Electron cyclotron heating and current drive $(\mathrm{ECH} / \mathrm{ECCD})$ is an attractive candidate for this purpose since the microwave power can be injected through a simple small launcher located far from the plasma surface. In addition, once the incident waves are mode-converted to electron Bernstein (EB) waves they can propagate into and cyclotron-heat the core plasma without the limit of the plasma cutoff density. This is particularly suitable for STs since they are essentially overdense.

In the Low Aspect ratio Torus Experiment (LATE) device, start-up and formation of spherical Tokamak by EB waves have been explored [5]. We expect two advantages by using EB waves for start-up compared with the conventional electromagnetic waves. The first one is based on the property that EB waves can have a high refractive index in parallel direction $\left(\mathrm{N}_{\|}\right)$more than 1 which the electromagnetic waves cannot. This property is particularly favourable for current ramp-up where a reverse voltage is exerted by self-induction since the forward momentum input to electrons upon the electron cyclotron resonance is proportional to $\mathrm{N}_{\|}$. The previous experiment [6] shows that EB waves can rapidly ramp-up the plasma current as fast as $\sim 260 \mathrm{kA} / \mathrm{s}$, comparable to the lower hybrid ramp-up rate, where a current carrying electron tail is developed against the reverse voltage from self-induction by a forward driving force on the tail via cyclotron absorption of high $\mathrm{N}_{\|} \mathrm{EB}$ waves. Another important property of EB waves is that there is no density limit for propagation and heating. This enables production and maintenance of plasmas at extremely overdense regime. In this paper we report a non-inductive start-up and formation of an extremely overdense ST plasmas by EB waves. The plasma current reaches up to $\sim 12 \mathrm{kA}$ and the electron density exceeds 7 times the plasma cutoff density, showing a highly overdense ST plasma can be produced and maintained solely by EB waves.

\section{Experimental setup}

The experiments are performed in the LATE device [7]. The vacuum vessel is a cylinder with a diameter of $1 \mathrm{~m}$ and a height of $1 \mathrm{~m}$ having a centre post for the toroidal field. There is no CS for the inductive current drive.

Three $20 \mathrm{~kW}$ and one $5 \mathrm{~kW}$ magnetrons at $2.45 \mathrm{GHz}$ are used for the experiment. The microwaves are launched through mid-plane launchers of open waveguide type with oblique angles as shown in Fig. 1. The transmission line on port $10 \mathrm{R}$ has a polarizer which can produce an arbitrary injection polarization [8].

\footnotetext{
${ }^{\mathrm{a}}$ Corresponding author: m-uchida@energy.kyoto-u.ac.jp
} 


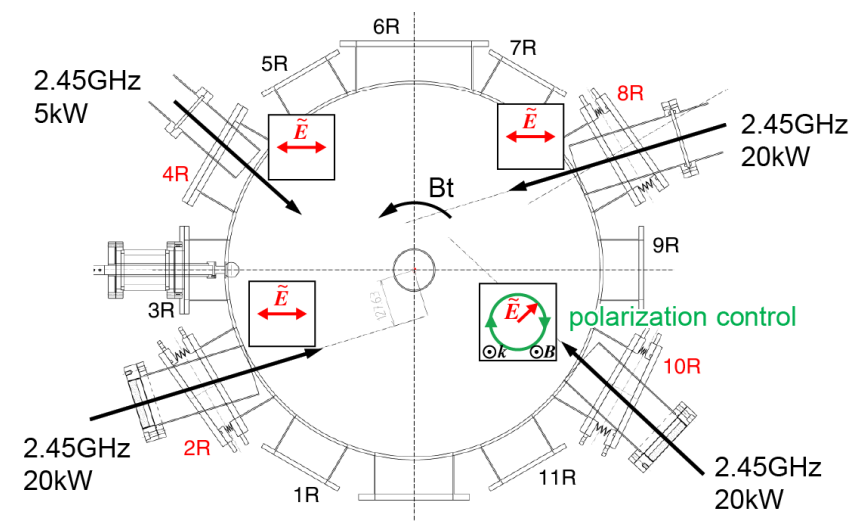

Figure 1. $2.45 \mathrm{GHz}$ microwave launchers.

Main diagnostics on LATE are seventeen flux loops and seven magnetic probes for magnetic analysis, 6 chords $70 \mathrm{GHz}$ interferometer system for line-integrated density measurement, extreme ultra violet (XUV) cameras with 20 vertical and 20 midplane chords, a fast CCD camera for visible plasma image, a spectrometer for visible light and an X-ray pulse height analysis system with four $\mathrm{Cd}-\mathrm{Te}$ detectors to measure photon energy spectra from the plasma in an energy range of $20-400$ $\mathrm{keV}$.

\section{Experimental results}

Figure 2 shows a typical discharge where the plasma current is started-up and ramped to $\sim 10.5 \mathrm{kA}$. The polarization of the injection waves are set to be the lefthand circularly polarized wave on Port $10 \mathrm{R}$ and the linearly polarized wave with the electric field vector on the mid plane on Port 2R, 4R and 8R, as shown in Fig. 1. Firstly a steady vertical field of $\mathrm{Bv}=20 \mathrm{G}(\mathrm{R}=25 \mathrm{~cm})$ is applied in addition to a steady toroidal field of $\mathrm{Bt}=0.072 \mathrm{~T}(\mathrm{R}=25 \mathrm{~cm})$ before the microwave injection. After the microwave power of $\mathrm{P} \sim 10 \mathrm{~kW}$ is injected the initial plasma is quickly produced at the fundamental ECR layer $\left(R_{E C R}=20.6 \mathrm{~cm}\right)$. After a while the plasma current start to flow and increases up to Ip $\sim 2 \mathrm{kA}$, resulting in the formation of closed flux surfaces under the steady vertical field [9]. Next, the plasma current is ramped up with ramps of the microwave power and the vertical field strength for equilibrium, finally reaches $\sim 10 \mathrm{kA}$ at $\mathrm{Bv}=120 \mathrm{G}$. The plasma is kept steady for
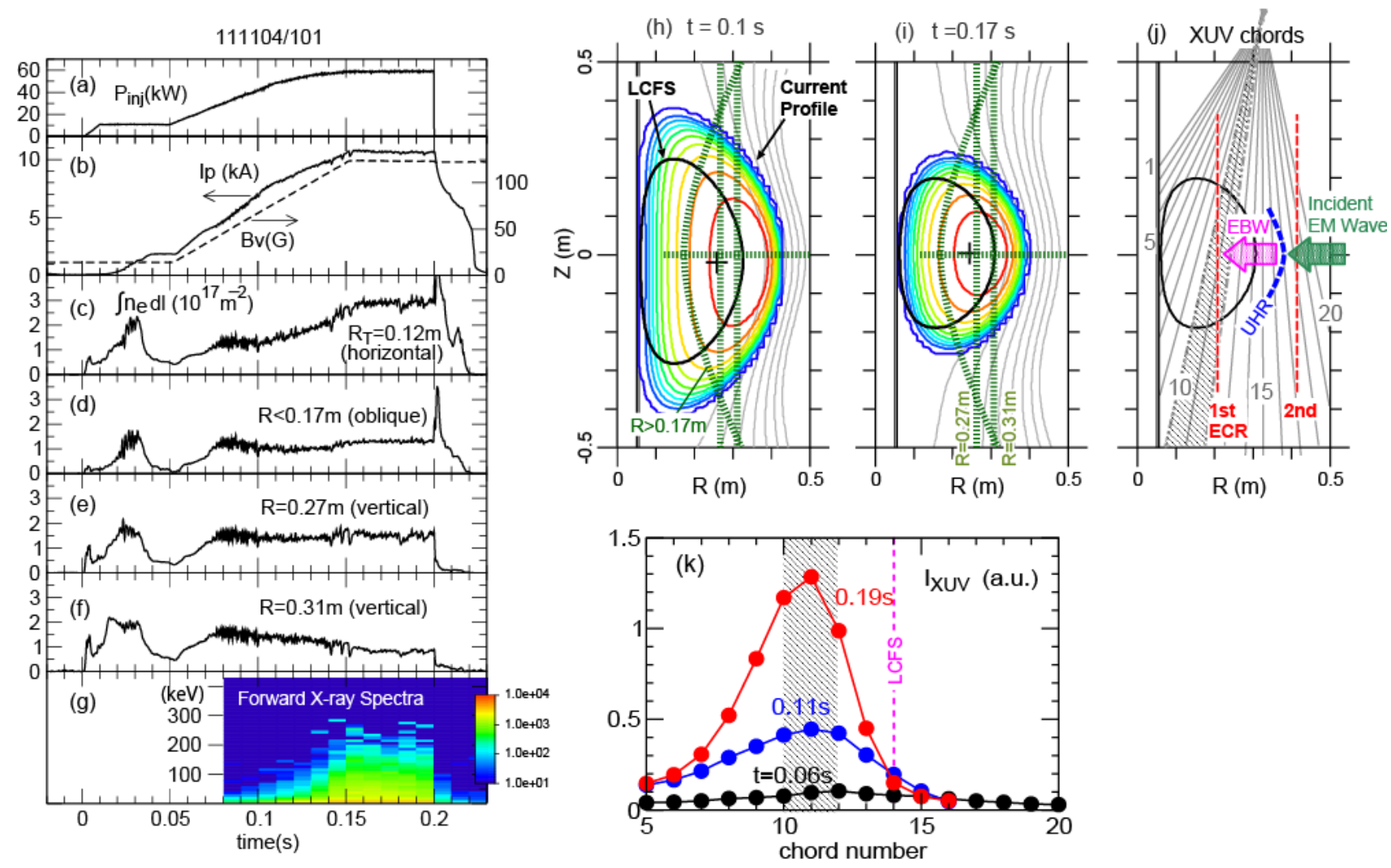

Figure 2. Typical discharge. (a) Injection power of $2.45 \mathrm{GHz}$ microwave, (b) Plasma current and vertical field strength at $\mathrm{R}=25 \mathrm{~cm}$, (c) Holizontal chord line-integrated density at a tangency radius of $\mathrm{Rt}=12 \mathrm{~cm}$, (d)-(f) Oblique and vertical chords line-integrated densities, (g) Forward X-ray emission from current carrying electrons measured along a tangency radius of Rt=25cm, (h) Current density and poloidal flux contours estimated from the magnetic analysis. Contours are equally spaced. Interferometer chords are also shown, (i) XUV chords and (k) XUV intensity profiles. 
$\sim 50$ ms until the microwave power is turned off.

The forward X-ray (Fig. 2(g)) measured along a tangency radius of $\mathrm{Rt}=25 \mathrm{~cm}$ develops both in energy range and photon counts as the plasma current increases, indicating that the current is carried by a fast electron tail which is driven by high $\mathrm{N}_{\|}$EB waves as described in the previous work [6]. Figure 2(h) and 2(i) show the plasma current profile and poloidal flux contours at $\mathrm{t}=0.1 \mathrm{sec}$ and $\mathrm{t}=0.17 \mathrm{sec}$, respectively, estimated from the magnetic analysis [6]. Since the typical energy of the tail electrons is $\sim 100 \mathrm{keV}$ and the plasma current is $10 \mathrm{kA}$ level, the current profile (i.e. orbits of the current carrying electrons) significantly shifts into the lower field side beyond the last closed flux surface (LCFS).

The multi-chords interferometer measurement of lineintegrated density (Figs. 2(c) - 2(f)) shows that the plasma is extremely overdense at the final stage of the discharge. The horizontal chord line-integrated density shown in Fig. 2(c) increases 2.5 times from $\mathrm{t}=0.1 \mathrm{~s}$ to $\mathrm{t}=0.17 \mathrm{~s}$, while its chord length inside the LCFS does not change as shown in Figs. 2(h) and 2(i). The lineaveraged electron density at the final steady phase $(\mathrm{t}=0.15-0.2 \mathrm{~s})$ reaches $\overline{\mathrm{n}}_{\mathrm{e}}=5.5 \times 10^{17} \mathrm{~m}^{-3}$, which is more than 7 times the plasma cutoff density. The lineintegrated densities on the other three chords (Figs. 2(d) 2(f)) do not change significantly during this interval, while their chord lengths inside the LCFS decrease as shown in Figs. 2(h) and 2(i). This also indicates that the density increases inside the LCFS.

In this discharge, the upper hybrid resonance (UHR) layer is estimated to lie to the slightly higher field side of the second harmonic ECR layer as shown in Fig. 2(j). In this case the incident microwaves are mode-converted to EB waves at the UHR layer in their first propagation band (between the fundamental and the second harmonic ECR layer). Then the EB waves propagate into the fundamental ECR layer and may heat the bulk electrons as well as the current carrying fast electrons in the plasma core. The time evolution of XUV emission profiles measured along the vertical chords (Fig. 2(j)) shows a significant increase near the fundamental ECR layer toward the final stage as shown in Fig. 2(k). The increment is much larger than the increments in vertical chords line-integrated densities (Figs. 2(d) - 2(f)), suggesting that the electron temperature increases at the plasma core. Impurity line radiation spectra taken every $50 \mathrm{~ms}$ from $\mathrm{t}=0.02 \mathrm{~s}$ (the measuring chord looks from $\mathrm{Rt}=18$ to $26.5 \mathrm{~cm}$ ) show that radiations at higher excitation energy such as $\mathrm{O}$ V $(72 \mathrm{eV})$ and $\mathrm{C} \mathrm{V}(304 \mathrm{eV})$ appears after $\mathrm{t}=0.12 \mathrm{~s}$ and significantly increases $(2-3$ times) toward the final stage. This also suggests that the electron temperature increases toward the final stage.



(g)

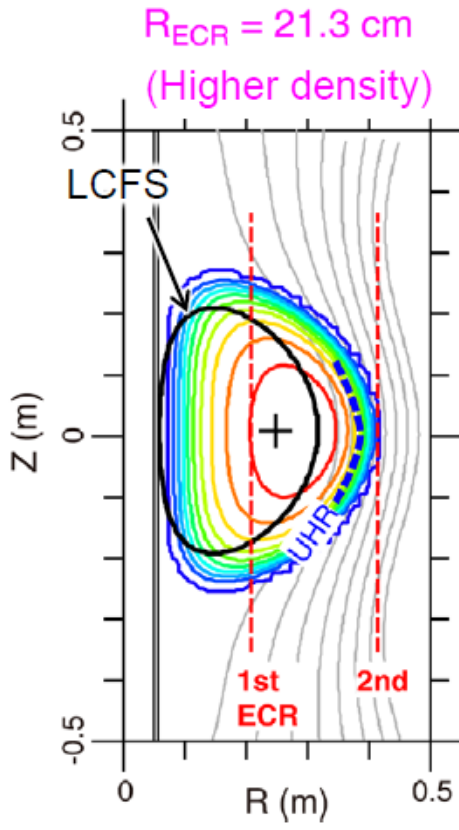

(h)

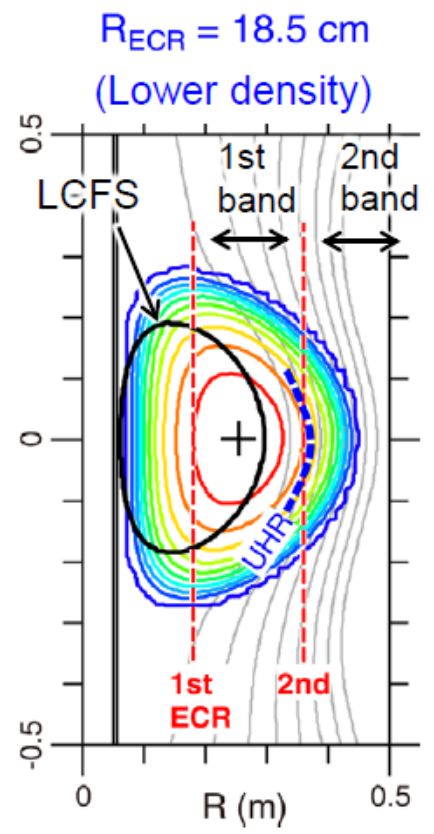

Figure 3. Discharges with $\mathrm{R}_{\mathrm{ECR}}=18.5 \mathrm{~cm}$ (blue) and $\mathrm{R}_{\mathrm{ECR}}=21.3 \mathrm{~cm}$ (magenta). Time traces of (a) injection power, (b) plasma current and vertical filed, (c) holizontal chord line-integrated density at a tangency radius of $\mathrm{Rt}=12 \mathrm{~cm}$, (d)-(f) oblique and vertical chord line-integrated density. Current density and poloidal flux contours with $(\mathrm{g}) \mathrm{R}_{\mathrm{ECR}}=18.5 \mathrm{~cm}$ and (h) $R_{\mathrm{ECR}}=21.3 \mathrm{~cm}$ at the final steady phase. 

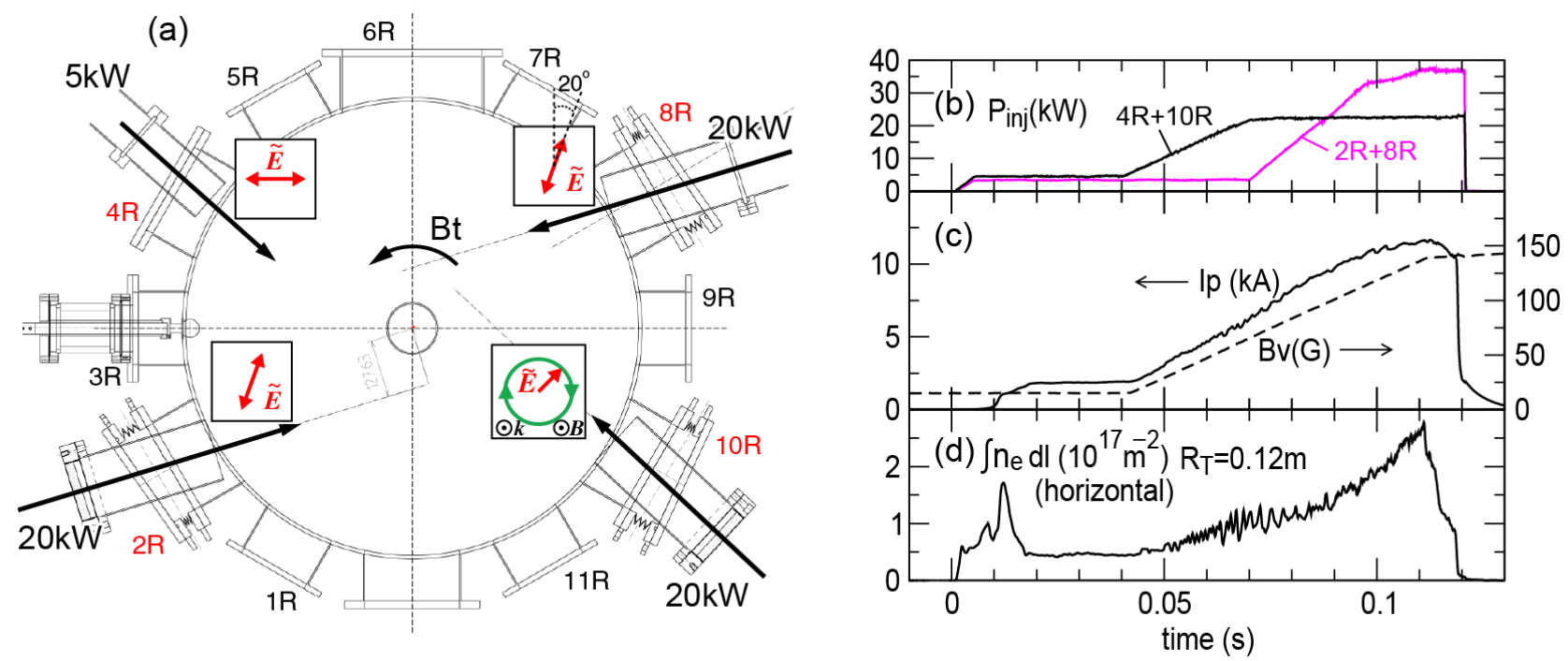

Figure 4. Time traces of (a) injection power of O-mode like polarization (black) and X-mode like one (red), (b) plasma current and vertical filed, (c) holizontal chord line-integrated density at a tangency radius of $\mathrm{Rt}=12 \mathrm{~cm}$. (d) Injection polarizations.

The good coupling to the EB waves at the first propagation band is of primary importance to obtain highly overdense plasmas. For reference, if we set the ECR layer at a slightly higher field side, the electron density becomes much lower and the current also lower. Figure 3 compares two discharges with $\mathrm{R}_{\mathrm{ECR}}=21.3 \mathrm{~cm}$ and $\mathrm{R}_{\mathrm{ECR}}=18.6 \mathrm{~cm}$ under the same injection power of $\sim 40 \mathrm{~kW}$ and the vertical field of $\mathrm{Bv}=105 \mathrm{G}$. While the density increases to $\overline{\mathrm{n}}_{\mathrm{e}} \sim 5 \times 10^{17} \mathrm{~m}^{-3}$ as the plasma current increases up to $\sim 9.3 \mathrm{kA}$ when $\mathrm{R}_{\mathrm{ECR}}=20.6 \mathrm{~cm}$, the density does not increase $\left(\bar{n}_{\mathrm{e}} \sim 1.6 \times 10^{17} \mathrm{~m}^{-3}\right)$ and the final current becomes lower $(\mathrm{Ip} \sim 8.5 \mathrm{kA})$ when $\mathrm{R}_{\mathrm{ECR}}=18.6 \mathrm{~cm}$. In the lower density case, the UHR is estimated to lie to the slightly lower field side on the mid plane as shown in Fig. 3(h). Therefore a large portion of the incident power may be mode converted to the EB waves at the second propagation band and absorbed before the 2nd harmonic ECR layer. This may lead to a significant reduction in the power coupled to the EB waves at the first propagation band, resulting in decreases in the density and the plasma current at the plasma core.

In the lower density case, the power deposition before the 2nd harmonic ECR layer may develop a group of energetic trapped electrons since the mirror ratio along the magnetic field line is very high outside the LCFS in the small aspect ratio configuration. The time evolutions of hard X-ray spectra measured along the vertical chord at $\mathrm{R}=33 \mathrm{~cm}$ and $\mathrm{R}=40.5 \mathrm{~cm}$ shows that the energy range and photon counts are much higher than the higher density case, suggesting the energetic trapped electrons develop outside LCFS in the lower density case. Their toroidal precession generates a low toroidal current as appeared as a large expansion of the plasma current profile beyond the LCFS as shown in Fig. 3(h).
In the higher density discharge, the density gradient near the UHR layer increases toward the final stage. For the discharge shown in Fig. 2 the density scale length become as low as $\operatorname{Ln} / \lambda_{0}=\mathrm{n} /\left(\lambda_{0} \mathrm{dn} / \mathrm{dr}\right) \sim 0.1$ at the final steady phase $\left(\lambda_{0}\right.$ is wavelength in free space and $n$ is the density). For this short density scale length, the polarization of the optimal injection mode to have the best mode conversion rate to EB waves become close to the X-mode as described in Ref. [10]. Then we need to adjust the polarization as the density increases during the discharge to keep the good mode conversion rate to EB waves. At the beginning of the discharge where the density is low and the density scale length is short, Omode like polarization has a high mode conversion rate, while X-mode like polarization become suitable at the final stage of the discharge. To study the polarization effect on the mode conversion to the EB waves, we have modified the polarizations on Port $2 \mathrm{R}$ and $8 \mathrm{R}$ using waveguide twists as shown in Fig. 4 (a). Among many attempts with combinations of the polarization and microwave power control, the largest plasma current and the highest density are obtained by injecting $\sim 40 \mathrm{~kW}$ microwave power with the X-mode like polarization and $\sim 20 \mathrm{~kW}$ power with the O-mode like polarization as shown in Fig. 4(b). The plasma current ramps up to 12 $\mathrm{kA}$ and the line-averaged density on the mid plane reaches $5.5 \times 10^{17} \mathrm{~m}^{-3}(\mathrm{~L}=0.55 \mathrm{~m}$ inside the LCFS), which is $\sim 7$ times the plasma cutoff density. Comparing with the result where all the power is injected with the O-mode like polarization (Fig. 1), the density is almost the same and the plasma current $20 \%$ higher. 
(e) (f) (g)

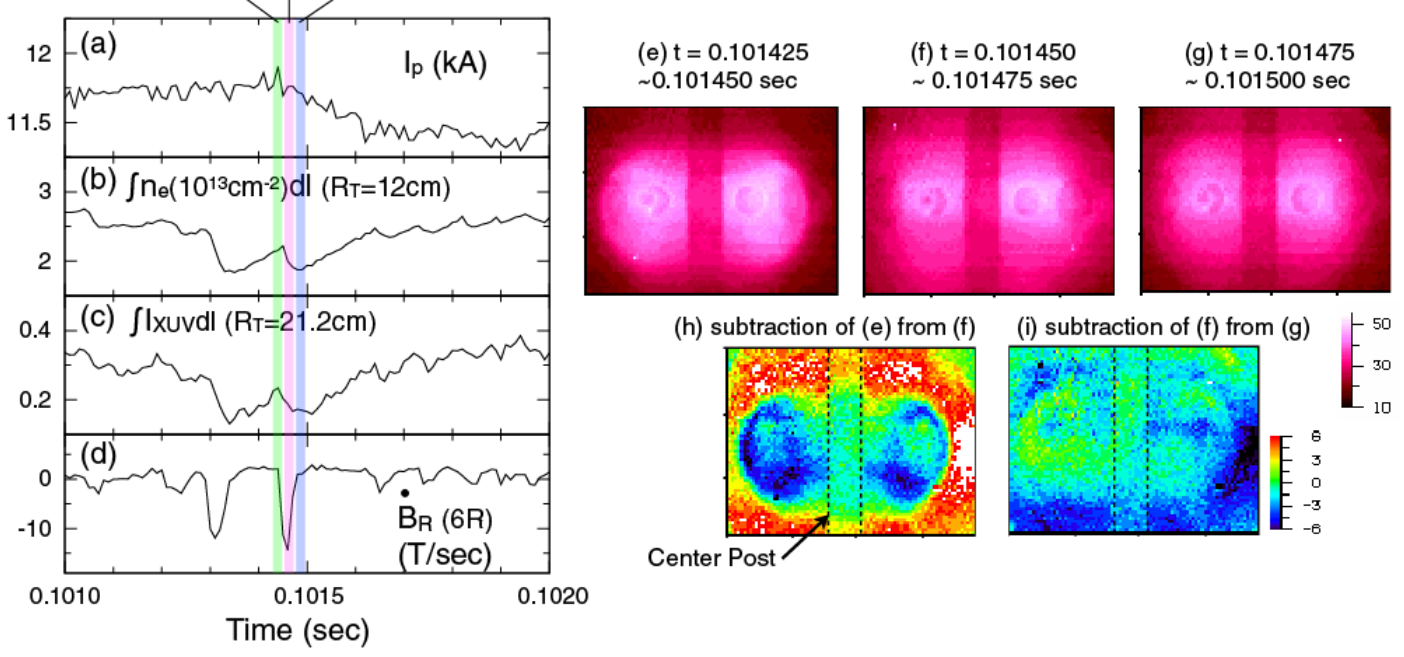

Figure 5. Plasma ejections across the LCFS are observed when Ip exceeds $10 \mathrm{kA}$. Time traces of (a) the plasma current, (b) horizontal chord line-integrated density, (c) XUV intensity $(\mathrm{Rt}=21.2 \mathrm{~cm})$ and $(\mathrm{d}) \mathrm{dBr} / \mathrm{dt}$ measuread by the magnetic on Port $6 \mathrm{R}$. (e)-(g) Visible camera images and (h)-(i) differences between two consecutive images.

In this discharge, however, the increment in the plasma current becomes slower after the plasma current exceeds $10 \mathrm{kA}$. In this stage, intermittent plasma ejections across the LCFS are observed as shown in Fig.5. These events are characterized by large spike signals on magnetic probes as shown in Fig. 5(d). The difference image of two consecutive visible camera images upon the event clearly indicates that the intensity decreases inside the LCFS and increases outside the LCFS (Figs. 5(e), (f) and $(\mathrm{h})$ ). The radial profile of horizontal XUV intensity on the mid plane also shows that the signal decreases inside the LCFS and increase outside the LCFS, and the inversion radius corresponds to the location of the LCFS. The line-integrated density in the horizontal chord decreases $\sim 40 \%$ on the largest drop and slowly recovers until the next event as shown Fig. 5(b). Repetition of such large ejection events causes a gradual decrease in the density and the plasma current. Suppression or mitigation of these events would be required to reach the higher density and higher current.

\section{Summary}

An extremely overdense special Tokamak plasma has been non-inductively started up and maintained for $50 \mathrm{~ms}$ solely by electron Bernstein (EB) wave heating and current drive in the LATE device. The plasma current reaches $12 \mathrm{kA}$ and the line-averaged electron density exceeds 7 times the plasma cutoff density with a 2.45 $\mathrm{GHz}$ microwave power of $60 \mathrm{~kW}$. Such a highly overdense plasma is obtained when a good coupling to the EB waves at their first propagation band is realized. The effect of the injection polarization on the mode conversion rate to $\mathrm{EB}$ waves at the extremely overdense regime has been investigated and an improvement in the plasma current is observed.

\section{References}

1. S. Nishio et al 2004 Proc. 20th Int. Conf. on Fusion Energy 2004 (Vilamoura, Portugal, 2004) (Vienna: IAEA)

2. K. Tobita et al, Fusion Eng. Des. 811151 (2006).

3. Y.-K. M. Peng et al, Plasma Phys. Control. Fusion, 47 (2005)

4. Y.-K. M. Peng et al, Proc. 23rd Int. Conf. on Fusion Energy 2010 (Daejeon, Korea, 2010) (Vienna: IAEA)

5. M. Uchida et al, 2012 Proc. 24th Int. Conf. on Fusion Energy 2012 (San Diego, USA, 2012) (Vienna : IAEA)

6. M. Uchida et al, Phys. Rev. Lett., 104065001 (2010)

7. T. Maekawa et al, Nucl. Fusion, 451439 (2005)

8. Y. Noguchi et al, Plasma Phys. Control. Fusion, 55 125005 (2013)

9. T. Yoshinaga et al, Phys. Rev. Lett., 96125005 (2006)

10. H. Igami et al, Plasma Phys. Control. Fusion, 48573 (2006) 